\title{
Temporal Plus Epilepsies: The Outcome after Non Invasive Presurgical Evaluation
}

\author{
Kchaou M $\mathbf{M}^{1,2^{*}}$, Ben Ali N ${ }^{1,2}$ and Belal $\mathbf{S}^{1,2}$ \\ ${ }^{1}$ Neurological department, Charles Nicolle Hospital, Boulevard du 9 Avril, Tunis 1006, Tunisia \\ ${ }^{2}$ Faculty of Medecine of Tunis, Tunisia
}

"Corresponding author: Mariem Kchaou, Neurological Department, Charles Nicolle Hospital, Boulevard du 9 Avril, Tunis 1006, Tunisia, Tel: 0021671562834; Fax: 0021671562834; E-mail: docmariem@yahoo.fr

Received date: May 19, 2015; Accepted date: July 17, 2015; Published date: July 21, 2015

Copyright: $\odot 2015$ Kchaou M et al. This is an open-access article distributed under the terms of the Creative Commons Attribution License, which permits unrestricted use, distribution, and reproduction in any medium, provided the original author and source are credited.

\begin{abstract}
Temporal plus epilepsies are a relatively frequent undiagnosed condition in temporal lobe epilepsies. We report two cases of temporal plus epilepsies suspected on ictal clinical and scalp-video EEG findings. No invasive EEG or functional imaging was performed since clinical, neuropsychological, neuroimaging data and video-EEG recordings are concordant. Our two patients underwent temporal lobectomy with an excellent outcome.
\end{abstract}

Keywords: Temporal plus epilepsies; Scalp EEG; Epilepsy surgery

\section{Introduction}

Temporal "plus" epilepsies $(\mathrm{T}+\mathrm{E})$ are a new described concept, accounting for $20 \%$ of all cases of temporal lobe epilepsies (TLE) [1]. The term "T+E" was used to unify some specific forms of multilobar epilepsies, which are characterized by: electro-clinical futures primarily suggestive of TLE, the absence of any detectable lesion on MRI with the exception of hippocampal sclerosis (HS), and EEG recordings demonstrating that seizures arise from a complex epileptogenic network including a combination of brain regions [2]. Seizures can involve the temporal lobe and the closed neighboured structures, such as the orbito-frontal cortex, the insula, the frontal, parietal operculum and the temporo-parieto-occipital junction [1-3].

Though the existence of $\mathrm{T}+\mathrm{E}$ has long been demonstrated by SEEG recordings, few published data on this topic exist making the identification of $\mathrm{T}+\mathrm{E}$ largely unknown [4]. Particularly, whether the diagnosis of $\mathrm{T}+\mathrm{E}$ can be suspected non-invasively, has not been well evaluated. We report 2 cases of patients suffering from medically intractable TLE with HS for whom the diagnosis of T+E was suspected. Clinical findings, video scalp EEG recordings, and the evolution after surgery are illustrated.

\section{Case Reports}

\section{Case 1:}

A 10 year-old, right-handed male had a history of epilepsy since 5 years of age. In his personal history, he experienced simple febrile seizure at the age of 14 months. Seizures were stereotyped and starting by an epigatric aura followed by loss of contact, motor and oroalimentary automatisms. These episodes lasted from 1 to $2 \mathrm{~min}$ without post ictal confusion or language deficit. The epilepsy was severe with daily seizures despite many antiepileptic drugs used including phenobarbital, carbamazepine and sodium valproate. Neurological examination was normal. Neuropsychological evaluation revealed visual memory impairment. Brain MRI showed a right HS.
Standard EEG recording showed frequent spikes and slow waves on the right temporal region (Figure 1).

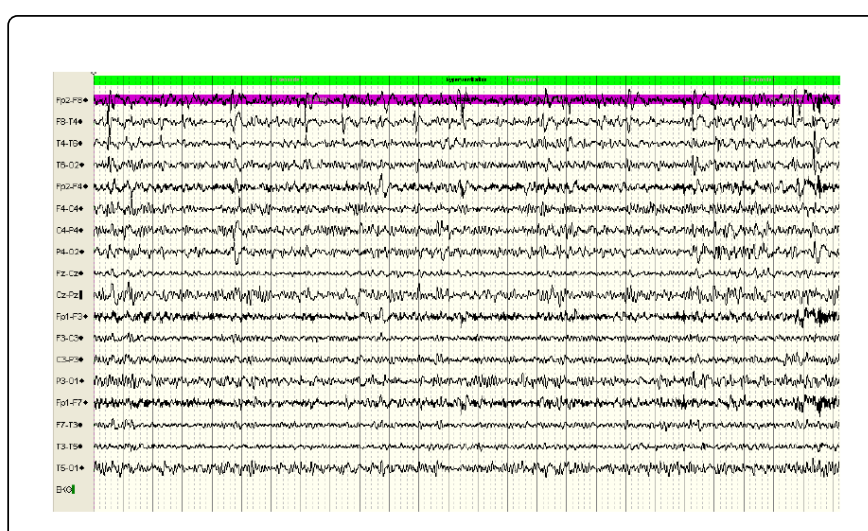

Figure 1: (Patient 1): Interictal EEG longitudinal montage: Frequent spikes and slow waves over the right temporal region.

On Video EEG monitoring, four ictal events were recorded. Seizure semiology started with a loss of contact, followed by a right deviation of the head and eyes, bilateral asymmetric tonic posturing during few seconds, with chewing and swallowing automatisms. No postictal language dysfunction was noted, the patient could name the objects given to him and replied correctly to physicians questions. Ictal EEG discharges were characterized by the disappearance of interictal EEG abnormalities and the occurrence of a rhythmic theta activity over the right anterior frontal region (FP2-F4), involving few seconds after the right anterior temporal region suggesting a right fronto-temporal onset of seizures (Figure 2). The electrographic seizure ended with an evident slowing, predominating over the frontal and right temporal region. Based on ictal clinical and video-EEG recording, the "epileptogenic zone" was defined as $\mathrm{T}+\mathrm{E}$, due to the fact that it included not only temporal lobe structures, but also the anterior frontal cortex. Surgery indication was considered. Further 
Citation: Kchaou M, Ben AN, Belal S (2015) Temporal Plus Epilepsies: The Outcome after Non Invasive Presurgical Evaluation. J Neurol

Page 2 of 4

investigations, such as invasive EEG or functional imaging, were not needed.

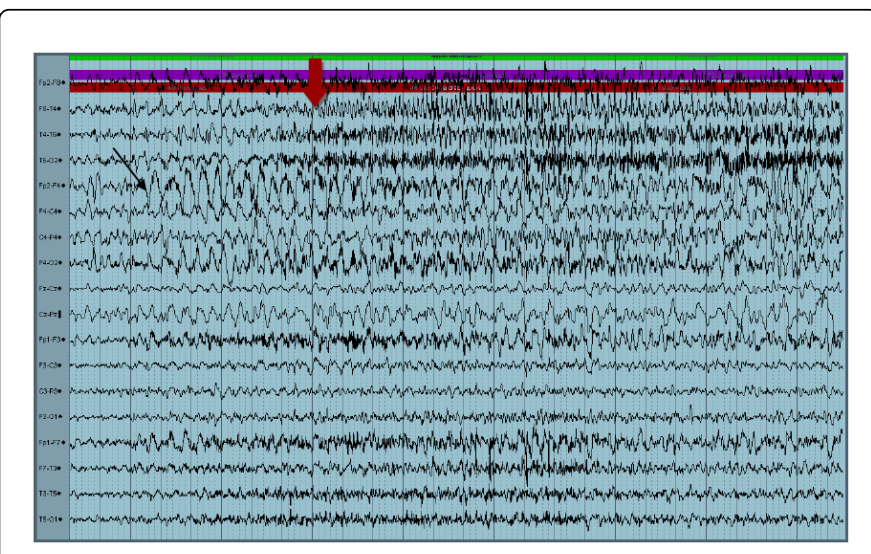

Figure 2: (Patient 1): Ictal EEG onset shows rhythmic theta activity over the right anterior frontal region (FP2-F4) (black arrow), involving few seconds later the right anterior temporal region (red arrow).

The patient underwent standard temporal lobectomy on September 2009. Pathological examination revealed severe HS. The child has remained seizure-free since the procedure. The neuropsychological assessment monitoring shows improvement in visual episodic memory. There were no visual changes reported by the patient and visual field examination was normal.

\section{Case 2:}

A 29-year-old, right-handed female admitted to our hospital for surgical evaluation of intractable epilepsy. In his personal history, she experienced an episode of complex febrile seizure at age of one year.

Since the age of 13, her seizures began with an epigastric aura followed by loss of consciousness, oroalimentary, motor, verbal automatisms and dystonic posturing of the right arm. Seizures occurred daily although good compliance and many antiepileptic drug trials. Neurological examination was normal. Neuropsychological evaluation was normal outside a depressive state. Brain MRI showed a left HS (Figure 3). Standard EEG recording showed frequent spikes on the left anterior temporal region (Figure 4).

During 4 days of video-EEG monitoring, three complex partial seizures were recorded. They consisted of a sudden cessation of ongoing activity, some left gestural automatisms and swallowing. Few seconds after, she had anxious expression on her face, fear and big anxiety. She could hide her face with her right arm then with the two arms, followed by a left deviation of the head and eyes then a hyper motor anarchic behavior especially involving the right upper limb. The postictal phase was characterized by nose-wiping, confusion with language deficit.

After analyses of the 3 montages, seizure onset on the scalp EEG recording consisted of rhythmic theta activity over the left frontal and anterior temporal region with a small spreading to the right hemisphere after few seconds of ictal onset. (Figure $5 \mathrm{a}, \mathrm{b}, \mathrm{c}$ and $\mathrm{d}$ ). Also in this case, "epileptogenic zone" was defined as $\mathrm{T}+\mathrm{E}$, because electric changes involved anterior fronto-temporal region. After correlation of clinical, neuropsychological, neuroimaging data and
video-EEG recording, surgery indication was considered. The patient underwent a left temporal lobectomy on May 2008 and has been seizure free from that date. Postoperative neuropsychological test showed persistent depression, verbal episodic memory impairment and language disturbance, which gradually recovered in 06 months. There were no changes in visual field.

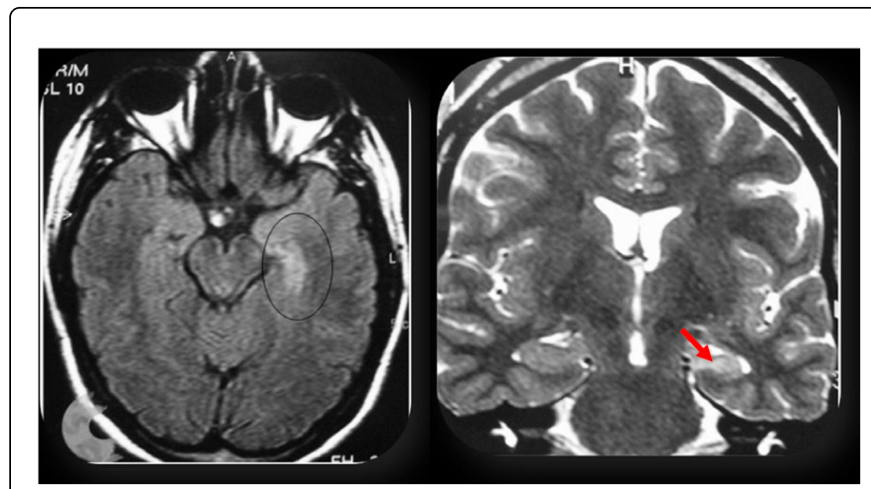

Figure 3: (Patient 2): Sagittal flair sequence and coronal T2 weighted image showing a left mesiotemporal sclerosis

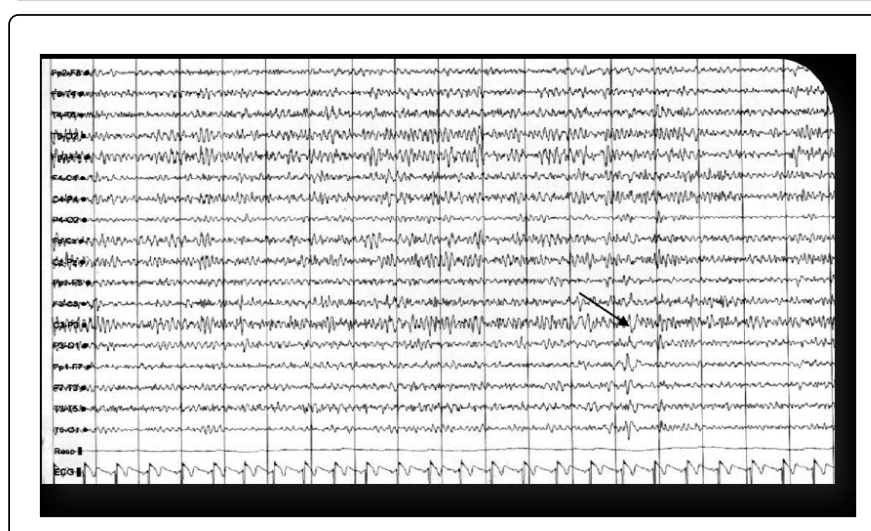

Figure 4: (Patient 2): Frequent interictal spikes arising from left anterior temporal region. 
Page 3 of 4

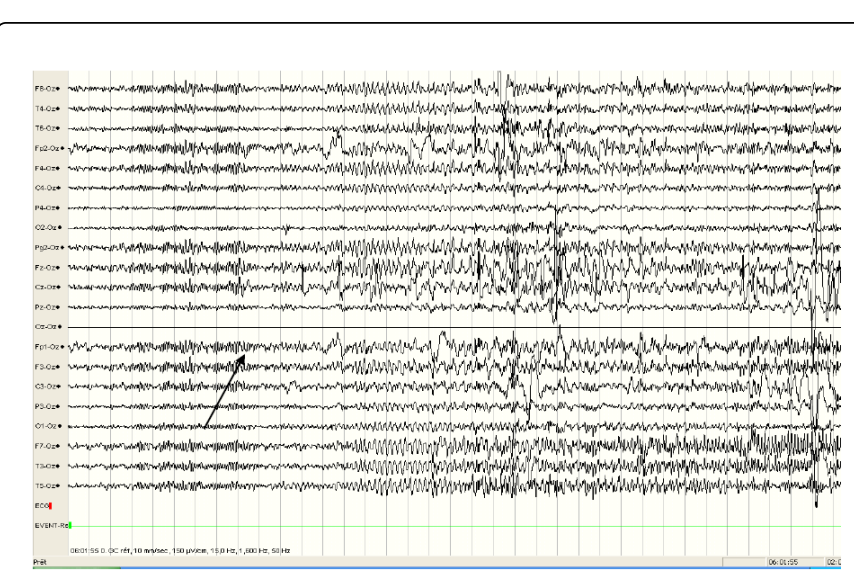

Figure 5a (Patient 2): Ictal onset in referential montage: lowvoltage fast activity ( 5 secondes) then, rhythmic theta activity predominating in the left regions, with a small spreading to the right side hemisphere.

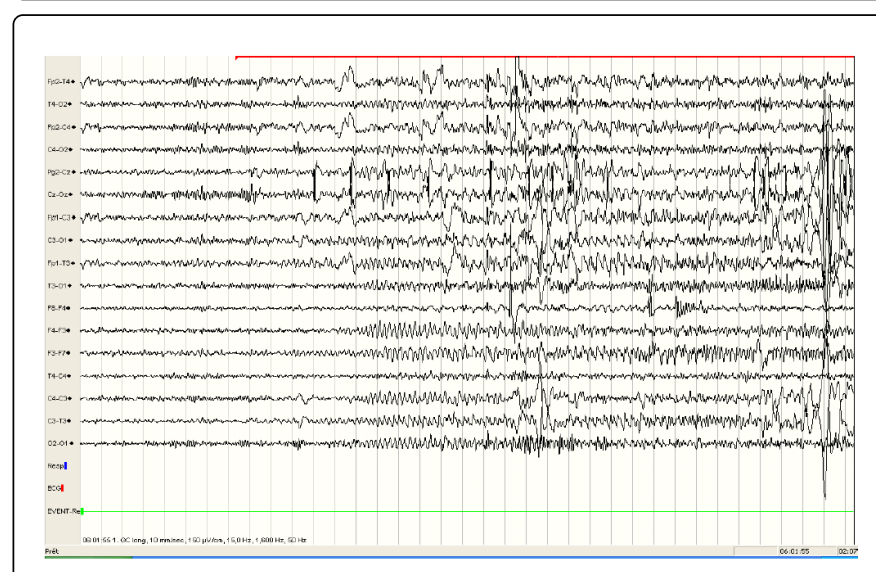

Figure 5b (Patient 2): Ictal onset in longitudinal montage: Showing well low- voltage fast activity in the left side, then rhythmic theta activity in the left fronto-temporal regions.

\section{Discussion}

We describe two patients, whom though the existence of HS on brain MRI, showed atypical electroclinical characteristics of TLE. On seizure semiology, the two patients could not warn at seizure onset. A large retrospective and comparative study of 80 patients (TLE. $n=58 ; T$ $+\mathrm{E} n=22$ ) had a proven diagnosis of seizure onset on the basis of stereotactic intracerebral EEG (SEEG) recordings. Patients with TLE presented more frequently an ability to warn at seizure onset than $\mathrm{T}+\mathrm{E}$ $(\mathrm{p}=0.003)$ [3]; Also, our two patients had motor manifestations consisting on tonic motor signs. These ictal symptoms are more associated with $\mathrm{T}+\mathrm{E}$ than TLE as they are mostly reported during frontal lobe seizures [3,5-7]. Moreover, versive manifestations of the eyes and head (patient 1) are usually in favour of the involvement of extratemporal localisation (frontal or posterior) [5,8]. Finally, Ictal EEG in our patients by showing first changes localized outside the borders of the TL over the frontal region are in favour of the diagnosis of $\mathrm{T}+\mathrm{E}$. Indeed, rhythmic spikes arose from the right anterior frontal region (FP2-F4) in patient 1. Also, the possible spreading of electric signs seen in patient 2 seems to be in favour of T+E than TLE [3].

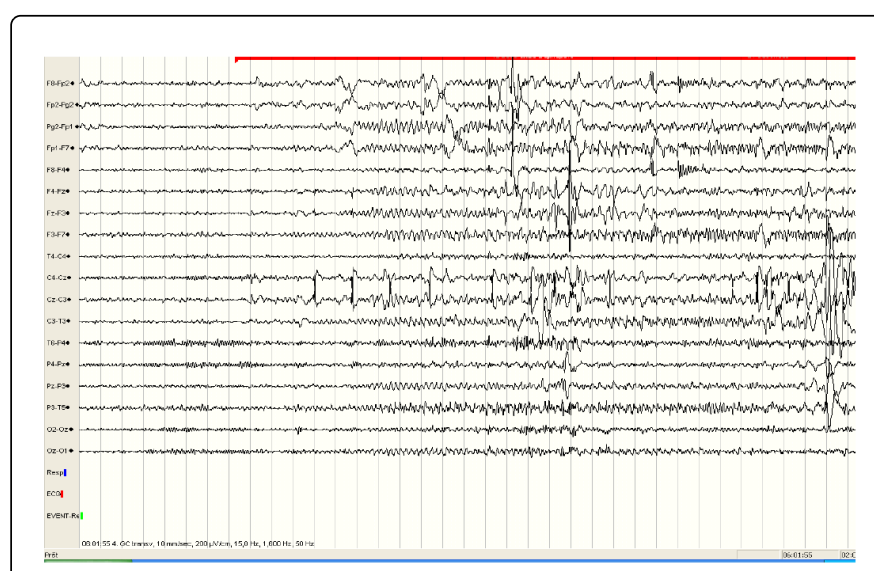

Figure 5c (Patient 2): Ictal onset in transversal montage: showing well rhythmic spikes arising from left fronto-temporal regions (FP1- F3).

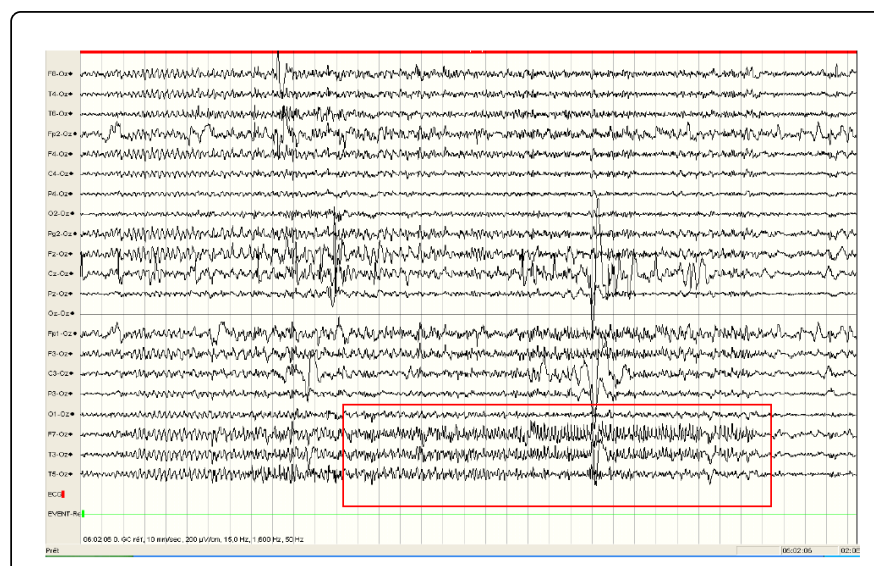

Figure 5d: (Patient 2): Rhythmic theta activity appears well in the left frontal and anterior temporal region.

The diagnosis of $\mathrm{T}+\mathrm{E}$ by non-invasive EEG recording, as in our two patients, is still the subject of many controversies [3]. For many authors, SEEG are mandatory and gives the opportunity to a more extensive cortectomy according to surgical possibilities and limits $[1,3,4]$. For others, the only video-scalp EEG recording is sufficient and able to perform successuful surgery. In fact, from 386 patients diagnosed with extratemporal refractory epilepsies, 61 were selected for resective surgery based on the presence of MRI lesions and concordant scalp recorded EEG data with a good outcome [9]. In developing country rare invasive recordings, exist, creating a gap in the identification and treatment of $\mathrm{T}+\mathrm{E}$. Our patients were selected based on an excellent analysis of scalp-video EEG recordings. Different findings were discussed between our team and the Neurophysiological Department of Charles Nicolle Hospital of Rouen through the EUMEDCONNECT Internet network project [10]. After a large temporal lobectomy, patients were seizure-free. Although not conclusive, these results can provide an additional argument for a successful surgery after non-invasive recordings in $\mathrm{T}+\mathrm{E}$ in a country with limited resources. 
Citation: Kchaou M, Ben AN, Belal S (2015) Temporal Plus Epilepsies: The Outcome after Non Invasive Presurgical Evaluation. J Neurol Neurophysiol 6: 302. doi:10.4172/2155-9562.1000302

Page 4 of 4

\section{References}

1. Ryvlin P, Kahane P (2005) The hidden causes of surgery-resistant temporal lobe epilepsy: extratemporal or temporal plus? Curr Opin Neurol 18: 125-127.

2. Kahane P, Barba C2, Rheims S3, Job-Chapron AS4, Minotti L5, et al (2015) The concept of temporal 'plus' epilepsy. Rev Neurol (Paris) 171 267-272.

3. Barba C, Barbati G, Minotti L, Hoffmann D, Kahane P (2007) Ictal clinical and scalp-EEG findings differentiating temporal lobe epilepsies from temporal 'plus' epilepsies. Brain 130: 1957-1967.

4. Haitao Zhu, Yong Liu, Yong Wu, Yingying Wang, Hongyi Liu, et al (2013) Temporal plus epilepsies: Electrophysiology studied with interictal magnetoencephalography and intracranial video-EEG monitoring. Seizure 22: 164-167.

5. Tassi L, Meroni A, Deleo F, Villani F, Mai R, et al. (2009) Temporal lobe epilepsy: neuropathological and clinical correlations in 243 surgically treated patients Epileptic Disorders 11: 281-292.
6. Prasad A, Pacia SV, Vazquez B, Doyle WK, Devinsky O (2003). Extent of ictal origin in mesial temporal sclerosis patients monitored with subdural intracranial electrodes predicts outcome. J Clin Neurophysiol 20: 243-248.

7. Manford M, Fish DR, Shorvon SD (1996) An analysis of clinical seizure patterns and their localizing value in frontal and temporal lobe epilepsies. Brain 119: 17-40.

8. Landré E, Trottier S, Turak B, Chassoux F, Devaux B(2003) Frontal lobe epilepsies: selection of surgical candidates Epileptic Disorders 5: 43-54.

9. Chaudhry N, Radhakrishnan A, Abraham M, Kesavadas C, Radhakrishnan VV, et al. (2010) Selection of ideal candidates for extratemporal resective epilepsy surgery in a country with limited resources. Epileptic Disord 12: 38-47.

10. Mrabet Khiari H, Khemiri E, Parain D, Hattab N, Proust F, et al. (2010) Epilepsy surgery program in Tunisia: an example of a Tunisian French collaboration. Seizure 19: 74-78. 